\title{
Examining the Dissociative Basis for Body Image Disturbances
}

\author{
Matthew Fuller-Tyszkiewicz (Corresponding author) \\ School of Psychology, Deakin University \\ 221 Burwood Highway, 3125, Melbourne, Australia
}

Tel: 61-3-9251-7344Ｅ-mail: matthewf@deakin.edu.au

\author{
Alexander Mussap \\ School of Psychology, Faculty of Health, Deakin University \\ 221 Burwood Highway, 3125, Melbourne, Australia \\ Tel: 61-3-9251-7103Ｅ-mail: mussap@deakin.edu.au
}

\begin{abstract}
Received: September 25, $2011 \quad$ Accepted: October 10, $2011 \quad$ Published: December 1, 2011
doi:10.5539/ijps.v3n2p3 URL: http://dx.doi.org/10.5539/ijps.v3n2p3
\end{abstract}

\begin{abstract}
Although dissociative symptoms have been linked with both food- and appearance-related aspects of eating disorders, the psychological mechanisms underlying these relationships remain unclear. The present study evaluated the hypothesis that the disturbances of self-identity attributed to dissociation can manifest as disturbances of body image and, in turn, undermine body-specific self-evaluations relevant to disordered eating (i.e., body comparison, body dissatisfaction, and internalization of the thin ideal). Ninety-three female university students completed self-report measures of dissociation and body-related aspects of disordered eating. In addition, the method of constant stimuli was used to experimentally derive three measures of body image disturbance: (1) accuracy of body size estimations (body image distortion), (2) ability to discriminate between different body sizes (body image sensitivity), and (3) consistency in one's body size estimations (body image variability). The findings show that dissociation is related to symptoms of disordered eating, and that these relationships may be mediated by body image instability. Collectively, these findings support the notion that the body image attitudes and behaviours that characterize eating disorders may derive from proprioceptive deficits due to dissociation.
\end{abstract}

Keywords: Dissociation, Body image, Body image instability, Women, Proprioception

\section{Introduction}

Dissociative disorders are characterized by deficits of consciousness, memory, and identity (American Psychiatric Association; APA, 2000), and are implicated in diminished behavioural self-control and disturbances of self-awareness (Brown, 2002; 2006). Dissociative symptoms are overrepresented in individuals diagnosed with an eating disorder (Demitrack et al., 1990; Groth-Marnat \& Michel, 2000; McManus, 1995; Waller, Ohanian, Meyer, Everill, \& Rouse, 2001). On this basis, it has been proposed that dissociative mechanisms may contribute to disordered eating symptoms (Everill \& Waller, 1995; Fuller-Tyszkiewicz \& Mussap, 2008). Unfortunately, the nature of this contribution remains unclear.

Dissociation is understood to be multidimensional in terms of its symptomatology and underlying psychological processes (Brown, 2002; Holmes et al., 2005), and may thus influence eating behaviours through its influences on body-specific behaviours, cognitions, and/or perceptions. Therefore, although there are numerous grounds for proposing a relationship between dissociation and ED symptoms (Everill \& Waller, 1995), the nature of this relationship is likely to be complex and difficult to characterize. The results of previous studies support this proposition in that two fundamentally different processes - behavioural disinhibition and cognitive suppression of potential threats - have been shown to serve as independent mediators of the relationship between dissociation and ED (Beato, Cano, \& Belmonte, 2003; Fuller-Tyszkiewicz \& Mussap, 2008, 2009; Hallings-Pott, Waller, Watson, \& Scragg, 2005). Moreover, it appears that the somatic manifestations of dissociation are most closely related to these ED symptoms (Fuller-Tyszkiewicz \& Mussap, 2008; Waller et al., 2003). The aim of the present 
study was to examine the relationship between body-specific symptoms of dissociation and body image, and to explore the implications of this relationship for ED symptomatology.

Body image is thought to reflect the accumulated integration of information from different sensory modalities (particularly visual and proprioceptive) and from multiple perspectives (i.e., from a front-on inspection in a mirror, or by looking down upon the visible parts of one's body with one's own eyes). In addition to such 'bottom-up' influences, cognitive and/or affective factors (such as mood states, memory, and the importance one attributes to appearance) are also thought to influence body image in a "top-down" fashion (Gardner \& Bokenkamp, 1996). These factors have been implicated in the fluctuations in body image (i.e., changes in self-perceived body size) observed in individuals over time (particularly when their body is being scrutinized; Cash \& Fleming, 2002), particularly in response to exposure to thin-idealized bodies (Irving, 1990), and in the distorted body image (i.e., systematic errors (usually over-estimations) in self-perceived body size) of individuals diagnosed with an ED (Bruch, 1962).

In recognition of the multidimensional nature of body image, and of the diverse approaches adopted by researchers wishing to evaluate body image (Smeets, 1997), the present study examined the relationship between dissociation and three different measures of body image disturbance: systematic errors in body size judgments (body image distortion), reduced sensitivity to body size differences (body image uncertainty), and variability over time in making body size judgments (body image instability). As discussed below, these three body image measures are thought to assess different body image processes.

On the basis of previous findings which demonstrate the link between cognitive-affective biases and body image distortion, it is thought that body image distortions reflect emotions, attitudes, and beliefs concerning appearance rather than the integrity of sensory processes related to body image integration (Gardner \& Bokenkamp, 1996). These cognitive-affective factors may influence body image formation by determining which sensory samples are integrated and their relative influence for current body image, which body components are the focus of the integration process, and the frequency with which the individual engages in this process. For example, an individual with negative body image may be more likely to attend to and integrate self-images that are unflattering (e.g., from particular postures), particularly with regard to areas of the body of cultural aesthetic significance (waist, stomach, thighs) (Mussap \& Salton, 2006). Alternatively, cognitive-affective factors may influence the availability of particular body image memories. For example, during negative mood states, the individual may be more likely to recall particularly unflattering/overweight representations of her/his body, which would in turn be more likely to impact negatively on self-esteem and increase the desire for body change (Smeets \& Kosslyn, 2001).

The performance of eating disordered (ED) individuals on attention-based tasks demonstrates their preferential processing of negative aspects of appearance and is consistent with the notion that these individuals have an attentional bias for negative and unflattering aspects of appearance (Shafran, Lee, Cooper, Palmer, \& Fairburn, 2007; Smeets, Roefs, van Furth, \& Jansen, 2008). While this selective attention may be due to their appearance concerns, the relation of dissociation to body-specific aspects of disordered eating as well as the role of dissociation for information processing raise the possibility that dissociative tendencies contribute to biases in body size judgments. Previous research has shown that individuals with heightened dissociative tendencies exhibit selective attention for threatening stimuli (Dorahy, Middleton, \& Irwin, 2005; Hermans, Nijenhuis, van Honk, Huntjens, \& van der Hart, 2006), and that this may extend to appearance-relevant information (Fuller-Tyszkiewicz \& Mussap, 2009). Accordingly, it is predicted in the present study that dissociative tendencies will be related to body image distortions towards over-estimation.

Body image uncertainty refers to an individual's threshold for detecting changes in her body size (Gardner \& Bokenkamp, 1996). This uncertainty is typically attributed to limitations in sensory processes involved in making size and shape judgments. The sensory processes are assumed to be visual rather than proprioceptive in that they are thought to undermine processing of the various "comparison" stimulus images presented to the individual during the course of the body image evaluation (usually body shapes or distorted photographs of their own body) rather than undermining processing of the individual's own body from a first-person perspective (the imagined "standard" stimulus).

A visual-sensory contribution to body image disturbances may, in theory, generalize to non-body shapes (indicative of a low-level sensory locus) or be specific to body shapes (indicative of a higher-level sensory locus). Although there are documented cases of functional blindness attributed to somatoform dissociative disorders (Bryant \& McConkey, 1999), these cases were characterised by either global blindness or uni-lateral blindness (as in hemi-neglect) rather than diminished visual acuity. Moreover, researchers have demonstrated that body 
image uncertainty is unrelated to disordered eating symptoms (Gardner \& Bokenkamp, 1996). Thus, while it is possible that dissociation contributes to body image uncertainty, extant literature does not support this link.

Body image instability reflects the extent to which an individual's body size estimates vary across time. There is a growing recognition that body image is not a stable construct and that this instability is most pronounced in individuals with elevated disordered eating symptoms (Mussap, McCabe, \& Ricciardelli, 2008; Rudiger, Cash, Roehrig, \& Thompson, 2007). Researchers have demonstrated that body size estimates vary as a function of mood (Baker, Williamson, \& Sylve, 1995), weight-related feedback (Mills \& Miller, 2007), time since last meal (Vocks, Legenbauer, \& Heil, 2007), and exposure to individuals with more attractive physiques (Haimovitz, Lansky, \& O'Reilly, 1993; Irving, 1990; van den Berg \& Thompson, 2007). Importantly, fluctuations in body size estimates have also been found in the absence of these cognitive-affective influences (e.g., Gardner \& Bokenkamp, 1996; Mussap et al., 2008), raising the possibility that body image instability is of relevance to ED symptoms.

Mussap and Salton (2006) suggest that individuals who are unable to maintain a stable body image may be more vulnerable to appearance concerns, direct criticism of their appearance, and perhaps even exposure to the thin ideal. Given the established link between dissociation and ED symptoms, as well as the assumed role of dissociation for undermining one's sense of self, Mussap and Salton contend that dissociative processes may be responsible for this variability in body size estimates. On the basis of this argument, the present study hypothesized that body image instability (as measured by assessing variability in body size judgments) would be positively related to dissociation and to the body-evaluative aspects of disordered eating (i.e., body comparison tendencies, body dissatisfaction, and internalization of the thin ideal). Moreover, it was anticipated that the relationship between dissociation and these body-evaluative variables would be mediated by body image instability.

\section{Method}

\subsection{Participants}

Participants were 93 women (age: $M=21.48, S D=3.44$ ), sampled from undergraduate classes at a metropolitan university in Melbourne, Australia. National Institute of Health (NIH) guidelines were applied to interpret body mass index (BMI) scores: $11.8 \%$ of this sample were "underweight" $(\mathrm{BMI}<18.5), 69.9 \%$ were "normal weight" (BMI 18.5-24.9), 17.2\% were "overweight" (BMI 25.0-29.9), and 1.1\% were "obese" (BMI $\geq 30.0)$. For the overall sample, average BMI was $22.04(S D=3.22)$.

\subsection{Materials}

Body dissatisfaction. The concern with body weight and concern with body shape subscales of the Eating Disorder Examination-Questionnaire (EDE-Q; Fairburn \& Beglin, 1994) were used to evaluate body dissatisfaction. These scales use 7-point Likert scales that range from $1=$ "No days" to $7=$ "Every day" to assess the number of days out of the last 28 that participants experience dissatisfaction with their appearance. Since we were interested in exploring the effects of overall body dissatisfaction (rather than the specific aspects of dissatisfaction), and since the measures of shape and weight concerns were multi-collinear $(r=.92)$, the two measures were averaged together to form a single measure of body dissatisfaction (DISS; $M=3.09, S D=1.58$ ). The body shape and weight subscales of the EDE-Q have good internal consistency and test-retest reliability, as well as concurrent and discriminant validity (Binford, LeGrange \& Jellar, 2005; Cooper, Cooper \& Fairburn, 1989; Mond, Hay, Rodgers, Owen \& Beumont, 2004).

Internalization. The Internalization-General subscale of the Sociocultural Attitudes Towards Appearance Questionnaire (SATAQ-3; Thompson, van den Berg, Roehrig, Guarda \& Heinberg, 2004) uses 5-point Likert scales that range from 0 (never) to 4 (always) to assess internalization of the thin ideal (INTERN; $M=2.82$, $S D=1.04)$. The SATAQ-3 has demonstrated content and convergent validity with regard to measures of body image and disordered eating, and it possesses adequate internal consistency (Thompson et al., 2004).

Body-based comparisons. The Physical Appearance Comparison Scale (PACS; Thompson, Heinberg \& Tantleff-Dunn, 1991) uses 5-point Likert scales that range from 0 (never) to 4 (always) to assess frequency of engagement in body comparisons (COMP; $M=2.88, S D=0.71$ ). The PACS measures frequency of comparison of one's whole body and one's body parts (i.e., stomach, hips, buttocks). The scale also measures frequency of comparison of one's body against the bodies of others (i.e., at parties, in magazines). An example item is "In social situations, I sometimes compare my figure to the figures of other people". The PACS has demonstrated internal consistency and test-retest reliability (Thompson et al., 1991). 
Somatoform dissociation. The Somatoform Dissociation Questionnaire (SDQ-20; Nijenhuis et al., 1996) uses 5 -point Likert scales that range from 0 (never) to 4 (always) to assess the presence and frequency of dissociative symptoms related to bodily sensations and functions. The SDQ-20 $(M=1.10, S D=.16)$ has good psychometric properties (Nijenhuis et al., 1996).

Body image. Participants were required to complete a computer-based task which assessed three key aspects of body image perception: (1) systematic distortions of perceived body size, which measures the extent to which an individual has the tendency to either over- or under-estimate the size of her own body, (2) body image uncertainty, which represents the individual's threshold for detection of computer-generated changes in her body size, and (3) body image instability, reflecting changes in body size estimates across blocks of trials.

Each participant was photographed with a digital camera by a research assistant who then uploaded this photograph to a desktop computer and digitally manipulated the image with Morphman ${ }^{\mathrm{TM}}$ software package to produce distorted versions of the original image. Distorted images were 4, 8, 12, 16 and 20\% narrower and wider than the original, thus producing 10 distorted images; 5 that were narrower and 5 that were wider than the original image.

Digital distortions were perpendicular to the primary axis of symmetry (i.e., vertical) for each body part, excluding head, feet, and hands. The resulting images were sufficiently naturalistic that distortion could not be identified on the basis of digital artefacts, such as poor resolution of images or disproportionate body part sizes. To further ensure that participants were responding to size distortions (rather than simply digital distortions), the original image was excluded from the computerized body image task.

The three body image measures were derived from participants' responses to the distorted images, as presented sequentially on a 17 " LCD computer monitor. Images were presented in blocks which involved 10 presentations of each of the 10 distorted images, in random order. Responses within a block were analysed by plotting the aggregate number of 'wider than me' responses as a function of body distortion level (see Mussap et al., 2008 for an example of this).

\subsection{Procedure}

Invitations to participate were announced at the beginning of lessons in various classes (sampled across several discipline areas) at a metropolitan university in Melbourne, Australia, and pamphlets were distributed to students in these classes. The pamphlets contained a plain language description of the study, and contact details through which they could obtain further information about the study. Participation was voluntary and anonymous (a random code was used to link responses for the questionnaire to responses to the experimental component of the study). Each participant received a $\$ 20$ gift card as an honorarium.

Each participant was tested individually in a laboratory setting, over a single session. Upon arrival, participants were instructed to remove bulky items of clothing which may obscure their actual body size and shape. They were given a knee-length, body-hugging white cotton t-shirt to wear for the photograph. Where necessary, pegs were placed at the back of the t-shirt to ensure that the clothing was tight fitting. Participants posed arms outstretched, standing upright in front of a black background. The photograph was taken and transferred to a desktop computer to be digitally manipulated by the experimenter. While the digitally distorted images were being created, participants filled out the questionnaire component of the study. Time taken by the experimenter to manipulate the digital image was approximately equal to the time required by participants to complete the questionnaire items.

After filling out the questionnaire, participants were seated in front of a 17" LCD computer monitor and informed that they would now be presented with a series of images of their own bodies, distorted to be wider or narrower than their actual body size. Participants were instructed to press the left button of a response pad when they thought that the presented image was thinner than their actual body size, and to press the right button when they thought that the image was wider than their actual size. To familiarize themselves with the task, participants were given a practice trial with all 10 distorted images $(-20,-16,-12,-8,-4,4,8,12,16,20 \%)$, presented in random order.

Participants completed three blocks of trials, each consisting of presentation of 10 distorted images, presented 10 times in random order. For each trial, the distorted image remained on the computer screen until the participants made their response. At the completion of each block, participants were permitted to take a break (of no more than 10 minutes duration), although they were not allowed to leave the testing area. This measure was taken in order to minimize contamination of body image measures (particularly body image instability) from cognitive and emotional fluctuations during the time of testing. 


\subsection{Data Analytic Strategy}

Path analyses were carried out to test the proposed influence of body image disturbances for the relationship between dissociation and body-specific evaluations relevant to disordered eating (i.e., body dissatisfaction, body comparison tendencies, and internalization of the thin ideal). To provide more accurate confidence intervals for estimated effects, the present study used a bias-corrected bootstrap re-sampling method tested through AMOS ${ }^{\mathrm{TM}}$ in preference for other statistical methods traditionally used to assess mediational effects (MacKinnon, Lockwood \& Williams, 2004; Shrout \& Bolger, 2002).

These pathways were tested if the following pre-conditions were met: (1) that the IV (i.e., somatoform dissociation) significantly correlated with the DV (i.e., body dissatisfaction, body comparison tendencies, or internalization of the thin ideal), (2) that the IV significantly correlated with the mediating variable (MV; i.e., body image instability), and (3) that the MV significantly correlated with the DV (Baron \& Kenny, 1986).

On the basis of these pre-conditions, bivariate correlations were conducted to test the relationships between somatic symptoms of dissociation, body image disturbances, and body-specific evaluations of appearance relevant to disordered eating (i.e., body comparison tendencies, body dissatisfaction, and internalization of the thin ideal).

\section{Results}

\subsection{Data Screening and Variable Construction Procedures}

\section{Data screening}

There was less than $2 \%$ missing data, and these cases were distributed randomly across items and participants. Missing values for items that comprise the EDE-Q subscales were replaced with a score of 0 ("No days"); missing values for the SDQ items were replaced with a "0" response (for the frequency of occurrence of the symptom). Subsequent data screening revealed that the dataset conformed to the assumptions of multivariate analysis (Curren, West, \& Finch, 1996; Tabachnick \& Fidell, 2005).

\section{Variable construction for components of body image}

Body image measures were calculated from each block of 100 trials (10 presentations of 10 levels of body distortion) by plotting the aggregate number of "wider than me" responses (made within a block) as a function of level of body distortion (see Figure 1 for an example). Weibull functions were then fitted to these data (examples of these fits are also shown in the figure), and from the function three points were extrapolated. The level of distortion corresponding to a "wider than me" response rate of $50 \%$ was taken to represent the point of subjective equality (PSE) - the level of distortion that corresponds to the participant's self-perceived body. The Weibull fits also allowed extrapolation of the levels of distortion that corresponded to "wider than me" response rates of $25 \%$ and $75 \%$. The distance between these two points was taken as the interval of uncertainty (IU), and half this distance was taken to represent the difference limen (IU/2), or level of body image uncertainty.

Based on these extrapolated points, three new body image variables were derived: (i) body image distortion ( $\mu \mathrm{PSE} ; M=3.56, S D=5.45$ ) was calculated as the average PSE of the three blocks of trials (positive values of $\mu \mathrm{PSE}$ indicate the presence of overestimation of body size); (ii) body image uncertainty ( $\mu \mathrm{IU} / 2 ; M=2.99, S D=.90$ ) was calculated as the average difference limen of the three pre-exposure blocks (a greater $\mu \mathrm{IU} / 2$ corresponds to greater insensitivity to differences in body size); and (iii) body image instability ( $\triangle \mathrm{PSE} ; M=2.12, S D=1.24$ ) was calculated as the standard deviation of PSE over the three blocks of trials. Inspection of the means of these body image measures reveals the presence of a significant body size overestimation, $t(92)=6.30, p<.01$ (one-sample t-test with a test value of 0 ).

\subsection{Relations between Components of Body Image, Dissociation, and Disordered Eating}

Table 1 displays the Cronbach's $\alpha$ and inter-relationships for the measures of dissociation, body image, and disordered eating administered in the present study. Cronbach's $\alpha$ is not reported for the averaged response to repeated use of a single item ( $\mu \mathrm{PSE}, \mu \mathrm{IU} / 2$, and $\Delta \mathrm{PSE})$. The remaining scales exhibited adequate reliability, with Cronbach's $\alpha>7$.

Consistent with previous findings (i.e., Beato et al., 2003; Fuller-Tyszkiewicz \& Mussap, 2008; Waller et al., 2003), the present study demonstrated that somatic symptoms of dissociation are positively associated with body-specific evaluations relevant to disordered eating (i.e., body comparison tendencies, body dissatisfaction, and internalization of the thin ideal). Table 1 also highlights the strong inter-relation of these various body-specific evaluations. Individuals who endorse sociocultural standards of appearance and/or who engage in body comparisons also tend to be dissatisfied with their appearance. 
As shown in Table 1, systematic errors (towards over-estimation) and temporal instability of body size judgments were both positively related to disordered eating symptoms. Interestingly, body image instability rather than distortion - tended to be more strongly related to the body-evaluative aspects of disordered eating (e.g., body dissatisfaction, appearance-based comparisons, and internalization of the thin ideal). Moreover, somatic symptoms of dissociation were related to temporal instability of body size judgments, but unrelated to systematic errors and uncertainty in body size estimates. Despite moderate to strong relationships with the other forms of body image disturbance, body image uncertainty was not reliably associated with symptoms of disordered eating.

\subsection{Path Analyses to Test Proposed Mediational Effects}

A series of path analyses were conducted to test the hypothesis that somatoform dissociation undermines the stability of one's internal representation of her body and, in turn, makes her vulnerable to unrealistic standards of appearance, to body-based comparisons and to appearance concerns. Consistent with prediction, significance testing of the indirect effects using AMOS ${ }^{\text {TM }}$ confirms that body image instability partially mediates the relationship between dissociation and body comparison $(\beta=.057, p<.01)$, the relationship with internalization of the thin ideal $(\beta=.038, p=.05)$, and the relationship with body dissatisfaction $(\beta=.048, p<.01)$. Figure 1 shows the standardized coefficients for these mediational pathways as well as the original standardized coefficients for the direct effect from somatoform dissociation to the body-evaluative variables (shown in brackets). Given that these mediational effects are partial, it is not surprising that the changes in coefficients are modest: body image instability accounts for approximately $22 \%$ of the shared relationship between dissociation and body comparison, $13 \%$ of the relationship between dissociation and internalization of the thin ideal, and $11 \%$ of the relationship between dissociation and body dissatisfaction.

\section{Discussion}

The primary aim of this study was to determine whether the disturbances of self-identity attributed to dissociation can manifest as disturbances of body image (i.e., distorted, uncertain, and/or unstable body image) which in turn undermine body-specific self-evaluations relevant to disordered eating (i.e., body comparison, body dissatisfaction, and internalization of the thin ideal).

The finding that somatic symptoms of dissociation were positively related to body image instability provides support for the proposition that dissociation undermines body image; that is, that the identity confusion attributed to dissociation can take the form of body identity confusion. Unlike previous investigations in which stability of body image was evaluated over relatively long time frames (e.g., across two weeks or more, Brinded, Bushnell, McKenzie, \& Wells, 1990), body image stability in the present study was evaluated across a single testing session. Although the use of a short time frame does not preclude the involvement of cognitive or affective processes, it does make it less likely that body image instability reflects fluctuations in these processes (i.e., it is unlikely that participants' mood or attitudes to appearance fluctuated substantially across blocks of trials within a single testing session). Rather, the relationship observed between dissociation and body image instability is more consistent with increased disturbances in the perceptual processes that are involved in maintaining body image.

Even if this interpretation is correct, the origin of the relationship is unlikely to be sensory (i.e., purely visual or proprioceptive). The reason for this is that dissociation was found to be unrelated to body image uncertainty, and it is uncertainty in making body size discriminations that is normally taken to reflect disturbances in underlying sensory processes (Gardner \& Bokenkamp, 1996). It is also unlikely that the relevance of body image instability is related to the body size distortions (usually over-estimations) that co-occur with ED (Bruch, 1962): The present study failed to find reliable associations between distortion and level of dissociation.

Consistent with findings from previous studies (i.e., Beato et al., 2003; Fuller-Tyszkiewicz \& Mussap, 2008), somatic dissociative experiences were positively related with body-evaluative aspects of disordered eating, such as body comparison tendencies, body dissatisfaction and internalization of the thin ideal. Path analyses were conducted to test the potential relevance of disturbances to body image processes for these relationships, and revealed small but significant indirect effects of body image instability for the relationship between dissociation and each of the body-evaluative variables. The finding that individuals with an unstable body image are more likely to engage in body comparisons is understandable, particularly if this instability undermines trust in one's self-evaluations. However, even if this interpretation is correct, it cannot readily explain why this confusion should lead to endorsement of the thin ideal and subsequent appearance concerns.

Importantly, these findings are not well-explained by other (and more popular) explanations of the relationship between dissociation and disordered eating symptoms either. According to cognitive suppression-based explanations (i.e., Heatherton \& Baumeister, 1991), heightened dissociative symptoms serve to predispose 
individuals to disengage from threatening stimuli. While this cognitive suppression has been demonstrated in individuals with an eating disorder when presented with general threats as well as appearance- and food-based threats (Hallings-Pott et al., 2005; Fuller-Tyszkiewicz \& Mussap, 2009; Meyer \& Waller, 1999; Waller \& Mijatovich, 1998), it seems unlikely that the highly variable body size estimates exhibited by individuals with dissociative symptoms was due to disengagement from the task. After all, random responses for the computerized body image task would likely result in inflated body image uncertainty scores, and yet individuals with heightened dissociative symptoms were no more likely in the present study to exhibit body image uncertainty than counterparts without dissociative symptoms.

Before concluding, it is important to consider the limitations of this study as these may impact upon present findings. Given the use of a cross-sectional study design, present results do not rule out the possibility of alternate models of the relationship between dissociation, body image, and disordered eating. Many theorists believe that body image disturbances found in ED populations are the result of cognitive-affective factors rather than perceptual disturbances. Proponents of this view may argue that body image instability is the consequence of, rather than underlying mechanism for, the relationship between dissociation and body-evaluative aspects of disordered eating. However, if this model were correct, we would also expect to find a correlation between dissociation and body image distortion, but we did not.

Most problematic is the difficulty inherent in attempting to isolate (i.e., measure independently) the emotional-cognitive, sensory, and perceptual contributors to body image through the use of psychophysical methods and analyses. The present study took several precautions to minimise contamination of cognitive-affective fluctuations for measurement of body image variables thought to reflect perceptual or integrative aspects of body image rather than cognitive-affective components. First, uncertainty was calculated indirectly, based on the pattern of findings for individual body size judgments. As such, it was not directly measured and, hence, less likely to be influenced by response biases, such as social desirability. Second, testing took place in a single session and blocks of trials were relatively short (compared with other body size estimation tasks), thus limiting the effects of cognitive-affective fluctuations and fatigue, respectively.

Given the emphasis in extant literature on the potential contribution of dissociation for disordered eating behaviours (particularly, binge-eating and purging), there is a paucity of explanatory models to account for the link found in previous studies between dissociation and body-evaluative aspects of disordered eating. The present findings provide support for the notion that dissociation undermines the perceptual processes that generate and maintain body image and, in turn, contribute to the body-specific aspects of disordered eating. While dissociative symptoms were positively related with body image instability, the relevance of temporal instability of body image for the body-evaluative aspects of disordered eating remains unclear. However, the present findings argue against the possibilities that unstable body image reflects disturbances to sensory systems (particularly, visual and proprioceptive) or to cognitive-affective biases which are thought to influence appraisals of one's own body. Future research is needed to confirm these findings and also to determine how body image instability may promote body dissatisfaction, appearance-based comparisons, and internalization of the thin ideal.

\section{References}

American Psychiatric Association. (2000). Diagnostic and statistical manual of mental disorders (4 ${ }^{\text {th }}$ ed. rev.). Washington, DC.

Baker, J.D., Williamson, D.A., \& Sylve, C. (1995). Body image disturbance, memory bias, and body dysphoria: Effects of negative mood induction. Behavior Therapy, 26, 747-759. http://dx.doi.org/10.1016/S0005-7894(05)80043-7

Baron, R.M., \& Kenny, D.A. (1986). The moderator-mediator variable distinction in social psychological research: Conceptual, strategic, and statistical considerations. Journal of Personality \& Social Psychology, 51, 1173-1182. http://dx.doi.org/10.1037/0022-3514.51.6.1173

Beato, L., Cano, T.R., \& Belmonte, A. (2003). Relationship of dissociative experiences to body shape concerns in eating disorders. European Eating Disorders Review, 11, 38-45. http://dx.doi.org/10.1002/erv.508

Binford, R.B., LeGrange, D., \& Jellar, C.C. (2005). Eating Disorders Examination versus Eating Disorders Examination-Questionnaire in adolescents with full and partial syndrome bulimia nervosa and anorexia nervosa. International Journal of Eating Disorders, 37, 44-49. http://dx.doi.org/10.1002/eat.20062 
Brinded, P.M.J., Bushnell, J.A., McKenzie, J.M., \& Wells, J.E. (1990). Body image distortions revisited: Temporal instability of body image distortion in anorexia nervosa. International Journal of Eating Disorders, 9 , 695-701. http://dx.doi.org/10.1002/1098-108X(199011)9:6<695::AID-EAT2260090612>3.0.CO;2-E

Brown, R.J. (2002). The cognitive psychology of dissociative states. Cognitive Neuropsychiatry, 7, 221-235. http://dx.doi.org/10.1080/13546800244000085

Brown, R.J. (2006). Different types of dissociation have different psychological mechanisms. Journal of Trauma and Dissociation, 7, 7-28. http://dx.doi.org/10.1300/J229v07n04_02

Bruch, H. (1962). Perceptual and conceptual disturbances in anorexia nervosa. Psychosomatic Medicine, 24, 187-194

Bryant, R.A., \& McConkey, K.M. (1999). Functional blindness: A construction of cognitive social influences. Cognitive Neuropsychiatry, 4, 227-241. http://dx.doi.org/10.1080/135468099395945

Cash, T.F., \& Fleming, E.C. (2002). Body image and social relations. In Cash, T.F., \& Pruzinsky, T. (Eds.). Body image: A handbook of theory, research and clinical practice. New York: Guilford Press, pp. 277-286.

Cooper, Z., \& Fairburn, C. (1987). The Eating Disorder Examination: A semi-structured interview for the assessment of the specific psychopathology of eating disorders. International Journal of Eating Disorders, 6, 1-8. http://dx.doi.org/10.1002/1098-108X(198701)6:1<1::AID-EAT2260060102>3.0.CO;2-9

Curran, P.J., West, S.G., \& Finch, J. (1996). The robustness of test statistics to non-normality and specification error in confirmatory factor analysis. Psychological Methods, 1, 16-29. http://dx.doi.org/10.1037/1082-989X.1.1.16

Demitrack, M.A., Putnam, F.W., Brewerton, T.D., Brandt, H.A., et al. (1990). Relation of clinical variables to dissociative phenomena in eating disorders. American Journal of Psychiatry, 147, 1184-1188

Dorahy, M.J., Middleton, W., \& Irwin, H.J. (2005). The effect of emotional context on cognitive inhibition and attentional processing in dissociative identity disorder. Behaviour Research and Therapy, 43, 555-568. http://dx.doi.org/10.1016/j.brat.2004.03.011

Everill, J.T., \& Waller, G. (1995). Dissociation and bulimia: Research and theory. European Eating Disorders Review, 3, 129-147. http://dx.doi.org/10.1002/erv.2400030303

Fairburn, C.G., \& Beglin, S.J. (1994). Assessment of eating disorders: Interview or self-report questionnaire? International Journal of Eating Disorders, 16, 363-370

Fuller-Tyszkiewicz, M., \& Mussap, A. (2008). The relationship between dissociation and binge eating. Journal of Trauma \& Dissociation, 9, 445-462. http://dx.doi.org/10.1080/15299730802226084

Fuller-Tyszkiewicz, M., \& Mussap, A.J. (2009). On the relationship between dissociation and binge eating. In F. Columbus (Ed.), Binge Eating (pp.1-11). NY: Nova Science Publishers, Inc.

Gardner, R.M., \& Bokenkamp, E.D. (1996). The role of sensory and nonsensory factors in body size estimations of eating disorder subjects. Journal of Clinical Psychology, 52, 3-15. http://dx.doi.org/10.1002/(SICI)1097-4679(199601)52:1<3::AID-JCLP1>3.0.CO;2-X

Groth-Marnat, G., \& Michel, N. (2000). Dissociation, comorbidity of dissociative disorders, and childhood abuse in a community sample of women with current and past bulimia. Social Behavior \& Personality, 28, 279-292. http://dx.doi.org/10.2224/sbp.2000.28.3.279

Hallings-Pott, C., Waller, G., Watson, D., \& Scragg, P. (2005). State dissociation in bulimic eating disorders: An experimental study. International Journal of Eating Disorders, 38, 37-41. http://dx.doi.org/10.1002/eat.20146

Heatherton, T.F., \& Baumeister, R.F. (1991). Binge eating as an escape from self-awareness. Psychological Bulletin, 110, 86-108. http://dx.doi.org/10.1037/0033-2909.110.1.86

Hermans, E.J., Nijenhuis, E.R.S., van Honk, J., Huntjens, R.J.C., \& van der Hart, O. (2006). Identity state-dependent attentional bias for facial threat in dissociative identity disorder. Psychiatry Research, 141, 233-236. http://dx.doi.org/10.1016/j.psychres.2005.03.007

Holmes, E.A., Brown, R.J., Mansell, W., Fearon, R.P., Hunter, E.C.M., Frasquilho, F., \& Oakley, D.A. (2005). Are there two qualitatively distinct forms of dissociation? A review and some clinical implications. Clinical Psychology Review, 25, 1-23. http://dx.doi.org/10.1016/j.cpr.2004.08.006 
Irving, L.M. (1990). Mirror images: Effects of the standard of beauty on the self- and body-esteem of women exhibiting varying levels of bulimic symptoms. Journal of Social and Clinical Psychology, 9, 230-242. http://dx.doi.org/10.1521/jscp.1990.9.2.230

MacKinnon, D.P., Lockwood, C.M., \& Williams, J. (2004). Confidence limits for the indirect effect: Distribution of the product and resampling methods. Multivariate Behavioural Research, 39, 99-128. http://dx.doi.org/10.1207/s15327906mbr3901_4

McManus, F. (1995). Dissociation and the severity of bulimic psychopathology among eating-disordered and non-eating-disordered women. European Eating Disorders Review, 3, 185-195. http://dx.doi.org/10.1002/erv.2400030307

Meyer, C., \& Waller, G. (1999). The impact of emotion upon eating behaviour: The role of subliminal visual processing of threat cues. International Journal of Eating Disorders, 25, 319-326. http://dx.doi.org/10.1002/(SICI)1098-108X(199904)25:3<319::AID-EAT10>3.0.CO;2-9

Mills, J.S., \& Miller, J.L. (2007). Experimental effects of receiving negative weight-related feedback: A weight guessing study. Body Image, 4, 309-316. http://dx.doi.org/10.1016/j.bodyim.2007.04.004

Mond, J.M., Hay, P.J., Rodgers, B., Owen, C., \& Beumont, P.J. (2004). Validity of the Eating Disorder Examination Questionnaire (EDE-Q) in screening for eating disorders in community samples. Behaviour Research \& Therapy, 42, 551-567. http://dx.doi.org/10.1016/S0005-7967(03)00161-X

Mussap, A.J., \& Salton, N. (2006). A 'rubber hand' illusion reveals a relationship between perceptual body image and unhealthy body change. Journal of Health Psychology, 11, 627-639. http://dx.doi.org/10.1177/1359105306065022

Mussap, A.J., McCabe, M.P., \& Ricciardelli, L.A. (2008). Implications of accuracy, sensitivity, and variability of body size estimations to disordered eating. Body Image, 5, 80-90. http://dx.doi.org/10.1016/j.bodyim.2007.07.003

Nijenhuis, E.R., Spinhoven, P., van Dyck, R., van der Hart, O., \& Vanderlinden, J. (1996). The development and psychometric characteristics of the Somatoform Dissociation Questionnaire (SDQ-20). Journal of Nervous \& Mental Diseases, 184, 688-694. http://dx.doi.org/10.1097/00005053-199611000-00006

Rudiger, J.A., Cash, T.F., Roehrig, M., \& Thompson, J.K. (2007). Day-to-day body-image states: Prospective predictors of intra-individual level and variability. Body Image, 4, 1-9. http://dx.doi.org/10.1016/j.bodyim.2006.11.004

Shafran, R., Lee, M., Cooper, Z., Palmer, R.L., \& Fairburn, C.G. (2007). Attentional bias in eating disorders. International Journal of Eating Disorders, 40, 369-380. http://dx.doi.org/10.1002/eat.20375

Shrout, P.E., \& Bolger, N. (2002). Mediation in experimental and nonexperimental studies: New procedures and recommendations. Psychological Methods, 7, 422-445. http://dx.doi.org/10.1037/1082-989X.7.4.422

Smeets, M.A. (1997). The rise and fall of body size estimation research in anorexia nervosa: A review and reconceptualization. European Eating Disorders Review, $\quad$ 5, 75-95. http://dx.doi.org/10.1002/(SICI)1099-0968(199706)5:2<75::AID-ERV190>3.0.CO;2-A

Smeets, M.A., \& Kosslyn, S.M. (2001). Hemispheric differences in body image in anorexia nervosa. International Journal of Eating Disorders, 29, 409-416. http://dx.doi.org/10.1002/eat.1037

Smeets, E., Roefs, A., van Furth, E., \& Jansen, A. (2008). Attentional bias for body and food in eating disorders: Increased distraction, speeded detection, or both? Behavioural Research and Therapy, 46, 229-238. http://dx.doi.org/10.1016/j.brat.2007.12.003

Thompson, J.K., Heinberg., L.J., \& Tantleff-Dunn, S. (1991). The Physical Appearance Scale (PACS). The Behavior Therapist, 14, 174

Thompson, J.K., van den Berg, P., Roehrig, M., Guarda, A.S., \& Heinberg, L.J. (2004). The sociocultural attitudes towards appearance scale-3 (SATAQ-3): Development and validation. International Journal of Eating Disorders, 35, 293-304. http://dx.doi.org/10.1002/eat.10257

Van den Berg, P., \& Thompson, J.K. (2007). Self-schema and social comparison explanations of body dissatisfaction: A laboratory investigation. Body Image, 4, 29-38. http://dx.doi.org/10.1016/j.bodyim.2006.12.004 
Vocks, S., Legenbauer, T., \& Heil, A. (2007). Food intake affects state body image: Impact of restrained eating patterns and concerns about eating, weight and shape. Appetite, 49, 467-475. http://dx.doi.org/10.1016/j.appet.2007.03.006

Waller, G., \& Mijatovich, S. (1998). Preconscious processing of threat cues: Impact on eating among women with unhealthy eating attitudes. International Journal of Eating Disorders, 24, 83-89. http://dx.doi.org/10.1002/(SICI)1098-108X(199807)24:1<83::AID-EAT7>3.0.CO;2-X

Waller, G., Ohanian, V., Meyer, C., Everill, J., \& Rouse, H. (2001). The utility of dimensional and categorical approaches to understanding dissociation in the eating disorders. British Journal of Clinical Psychology, 40, 387-397. http://dx.doi.org/10.1348/014466501163878

Waller, G., Babbs, M., Milligan, R., Meyer, C., Ohanian, V., \& Leung, N. (2003). Anger and core beliefs in the eating disorders. International Journal of Eating Disorders, 34, 118-124. http://dx.doi.org/10.1002/eat.10163

Table 1. Bivariate correlations (Pearson's $r$ values are reported)

\begin{tabular}{|ll|l|l|l|l|l|l|}
\hline & & SDQ & DIS & COM & INT & $\mu \mathrm{PSE}$ & $\mu \mathrm{IU} / 2$ \\
\hline 1. & SDQ & $\mathbf{. 8 7}$ & & & & & \\
\hline 4. & DISS & .44 & $\mathbf{. 9 5}$ & & & & \\
\hline 7. & COMP & .27 & .70 & $\mathbf{. 8 6}$ & & & \\
\hline 8. & INTERN & .31 & .63 & .77 & $\mathbf{. 9 3}$ & & \\
\hline 9. & $\mu \mathrm{PSE}$ & .05 & .26 & .13 & .11 & $\mathbf{n a}$ & \\
\hline 10. & $\mu \mathrm{IU} / 2$ & -.04 & .09 & .03 & .03 & .76 & na \\
\hline 11. & $\Delta \mathrm{PSE}$ & .22 & .30 & .31 & .23 & .31 & .33 \\
\hline
\end{tabular}

Notes: All bivariate correlations greater than .20 are significant at $\mathrm{p}<.05$, na $=$ not applicable. The numbers presented in bold along the main diagonal represent internal consistency values (i.e., Cronbach's $\alpha$ ) for these variables. SDQ $=$ somatoform dissociative experiences, $\mathrm{DISS}=$ body dissatisfaction, $\mathrm{COMP}=$ body-based comparison tendencies, INTERN = internalization of the thin ideal, $\mu \mathrm{PSE}=$ average body size distortion, $\mu \mathrm{IU} / 2$ $=$ average body image uncertainty, and $\triangle \mathrm{PSE}=$ body image instability. 

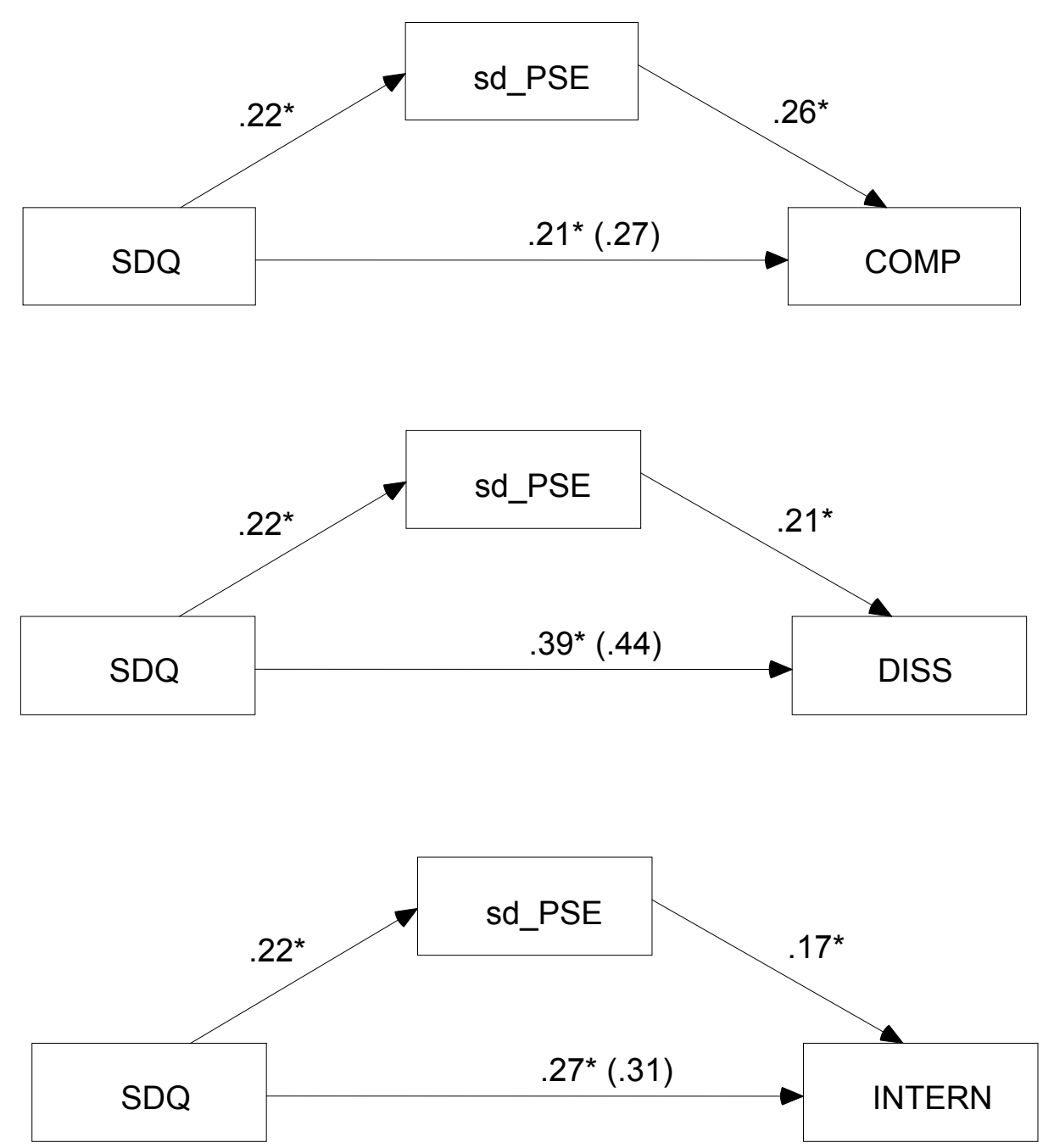

Notes: ${ }^{*} \mathrm{p}<.05$. Numbers shown in the figure represent standardized coefficients. The standardized coefficients for the direct path from IV to DV are presented in brackets. SDQ = somatoform dissociation, sd_PSE = body image instability, COMP = body comparison tendencies, DISS = body dissatisfaction, and INTERN = internalization of the thin ideal.

Figure 1. Mediational influence of body image instability for the relationships between dissociation and the body-evaluative aspects of disordered eating 